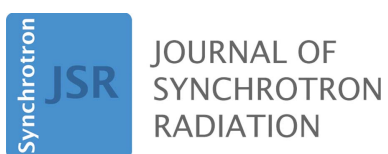

ISSN 1600-5775

Received 25 April 2017

Accepted 19 June 2017

Edited by G. Grübel, HASYLAB at DESY, Germany

Keywords: free-electron laser; higher-harmonic oscillation; bunch length; detuning curve.

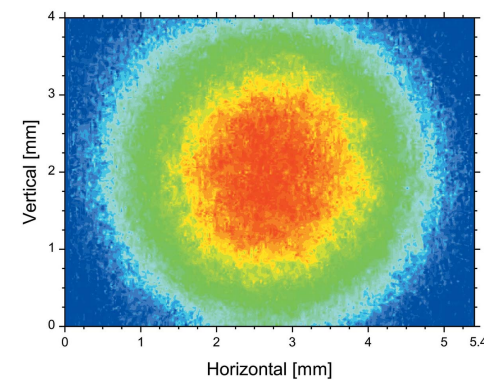

OPEN $\odot$ ACCESS

\section{Electron-bunch lengthening on higher-harmonic oscillations in storage-ring free-electron lasers}

\author{
Norihiro Sei, ${ }^{a *}$ Hiroshi Ogawa ${ }^{a}$ and Shuichi Okuda ${ }^{b}$

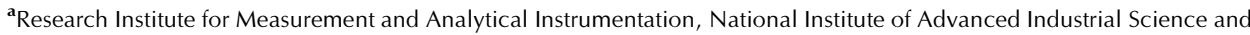 \\ Technology, 1-1-1 Umezono, Tsukuba, Ibaraki 305-8568, Japan, and ${ }^{\mathbf{b}}$ Quantum and Radiation Engineering, Osaka \\ Prefecture University, 1-1 Gakuen-cho, Sakai, Osaka 599-8531, Japan. *Correspondence e-mail: sei.n@aist.go.jp
}

The influence of higher-harmonic free-electron laser (FEL) oscillations on an electron beam have been studied by measuring its bunch length at the NIJI-IV storage ring. The bunch length and the lifetime of the electron beam were measured, and were observed to have become longer owing to harmonic lasing, which is in accord with the increase of the FEL gain. It was demonstrated that the saturated FEL power could be described by the theory of bunch heating, even for the harmonic lasing. Cavity-length detuning curves were measured for the harmonic lasing, and it was found that the width of the detuning curve was proportional to a parameter that depended on the bunch length. These experimental results will be useful for developing compact resonator-type FELs by using higher harmonics in the extreme-ultraviolet and the $\mathrm{X}$-ray regions.

\section{Introduction}

The generation of higher-harmonic oscillation is an effective technique for shortening the wavelength of a free-electron laser (FEL) oscillation. Because this technique requires an optical cavity, the working region of the wavelength of the higher-harmonic oscillation is limited by the performance of the optical cavity. However, pioneering studies on higherharmonic FEL oscillations have made significant advances since the dawn of the development of FEL devices (Colson, 1981; Jerby \& Gover, 1986). A third-harmonic FEL oscillation was achieved for the first time with a linear accelerator during the 1980s (Benson \& Madey, 1989), and over the years several groups have achieved FEL oscillations on seventh or lower harmonics (Warren et al., 1990; Kato et al., 1998; Neil et al., 2001; Hajima et al., 2001; Wu et al., 2008; Sei et al., 2012a). Recently, the technique of higher-harmonic oscillation has been examined for its application in an X-ray FEL oscillator (Dai et al., 2012), which is based on an advanced energyrecovery linear accelerator and is equipped with a highreflectivity crystal in the X-ray cavity (Kim et al., 2008). By utilizing this technique, the FEL oscillation in the X-ray region can be realised using an electron beam with a relatively low energy of $3.5 \mathrm{GeV}$ (Deng \& Dai, 2013).

Previously, we have carried out advanced studies to determine the characteristics of higher-harmonic FEL oscillations using the infrared FEL system of the NIJI-IV storage ring (Yamazaki et al., 1998; Sei et al., 2009). We demonstrated the generation of FEL oscillations using up to the seventh harmonic (Sei et al., 2012b), which is the highest harmonic order ever achieved in the field of higher-harmonic FEL oscillations. In addition, it was demonstrated that the higher harmonic was useful for shortening the FEL wavelength using 
the higher interference of the dielectric multilayer mirrors in the optical cavity (Sei et al., 2012c). Several aspects of the FEL gain for the higher-harmonic FEL oscillations were clarified in detail by the experiments performed with the NIJI-IV infrared FEL system (Sei et al., 2010, 2014b).

However, systematic studies on the influence of higherharmonic FEL oscillations on the electron beam have not been reported. It is important for the development of the beam physics to investigate the behaviour of the electron beam on the higher-harmonic FEL oscillation. The storagering FEL oscillation causes bunch lengthening of the electron beam via energy spreading (Renieri, 1979). Thus, we observed the bunch length of the electron beam on higher-harmonic FEL oscillations by direct and indirect methods. It was clarified that the behaviour of the electron beam on higherharmonic FEL oscillation could be described using the theory of bunch heating in the storage ring (Elleaume, 1984a). In this article we report on a variety of such observational results of the electron beam on the higher-harmonic FEL oscillation using the NIJI-IV infrared FEL system.

\section{Observation of bunch lengthening with a dual streak camera}

The FEL receives energy from the electron beam while interacting with it and causes a spread in the energy of the electron beam. In the case of a storage-ring FEL, the relative energy spread $\sigma_{\gamma} / \gamma$ has the following relationship with the bunch length of the electron beam $\sigma_{1}$ (Wiedemann, 1995),

$$
\sigma_{1}=\frac{\alpha c}{\Omega_{\mathrm{s}}}\left(\frac{\sigma_{\gamma}}{\gamma}\right)
$$

where $c$ is the speed of light, $\alpha$ is the momentum compaction factor, and $\Omega_{\mathrm{s}}$ is the angular synchrotron frequency. As is evident from equation (1), an increase in the energy spread due to the FEL oscillation leads to an increase in the bunch length (Roux et al., 1997). Because the FEL gain is inversely proportional to the bunch length, the bunch lengthening suppresses the amount of FEL oscillation. Furthermore, an increase in the energy spread causes an increase in the electron-beam size at positions in the storage ring that have a large dispersion function. However, the dispersion function is almost zero along the two long straight sections, where each optical klystron is installed in the NIJI-IV (Sei et al., 2003). For these two regions, the FEL gain is not affected by an increase in the beam size on the FEL oscillation. Therefore, the saturation of the FEL power is caused by an increase of the energy spread and the bunch length of the FEL systems in the NIJI-IV. When the power of the storage-ring FEL saturates, the FEL gain on the $n$th harmonic $G_{n}$ is equal to the loss of the optical cavity $l_{\mathrm{c}}$. According to the one-dimensional FEL theory on the bunch heating process using the optical klystron (Elleaume, 1984a), the following approximation for the bunch length holds true (Sei et al., 2003),

$$
\frac{G_{n}}{l_{\mathrm{c}}}=\frac{\left[\sigma_{1}\right]_{\mathrm{ON}}\left[f_{\gamma n}\right]_{\mathrm{OFF}}}{\left[\sigma_{1}\right]_{\mathrm{OFF}}\left[f_{\gamma n}\right]_{\mathrm{ON}}}
$$

where the suffixes 'ON' and 'OFF' denote the state of the FEL oscillation (Billardon et al., 1985); namely, $\left[\sigma_{1}\right]_{\mathrm{ON}}$ and $\left[\sigma_{1}\right]_{\mathrm{OFF}}$ are the laser-on and laser-off bunch lengths, respectively. The modulation factor for the $n$th harmonic $f_{\gamma n}$ is given by

$$
f_{\gamma n}=\exp \left[-8 \pi^{2} n^{2}\left(N_{\mathrm{u}}+N_{\mathrm{d}}\right)^{2}\left(\sigma_{\gamma} / \gamma\right)\right]
$$

where $N_{\mathrm{u}}$ is the number of periods in one undulator section of the optical klystron and $N_{\mathrm{d}}$ is the number of periods of the fundamental wavelength passing over an electron in the dispersive section (Elleaume, 1984b). By using equation (1), equation (3) can be described using the bunch length in substitution for the energy spread. It is possible to quantitatively evaluate the influence of the FEL oscillation on the electron beam by observing the influence of the bunch length on the FEL oscillation.

Then, we observed the synchrotron radiation from a bending magnet with a dual-sweep streak camera of $2 \mathrm{ps}$ resolution and measured the bunch lengths on the fundamental and third-harmonic FEL oscillations (Sei et al., 2008). The electron energy in the infrared FEL experiments operated in single electron-bunch mode was $310 \mathrm{MeV}$. The relative energy spread of the electron bunch was $4.0 \times 10^{-4}$ in the single-bunch mode, and the bunch length, which was independent of the electron-beam current below $5 \mathrm{~mA}$, was approximately 90 ps (Sei et al., 2009). Dielectric multilayer mirrors having target wavelengths of $870 \mathrm{~nm}$ were used in the experiments (Sei et al., 2010). As shown in Fig. 1, the higherharmonic FEL whose profile was almost a perfect TEM $_{00}$ mode was obtained in the NIJI-IV infrared FEL system. Table 1 shows the parameters of the optical klystron ETLOKIII and the values of the saturated FEL power derived from the higher-harmonic FEL experiments. The deflection parameter of the ETLOK-III (Sei et al., 2002), K, was 2.09 and 4.14 for the fundamental and the third-harmonic FELs, respec-

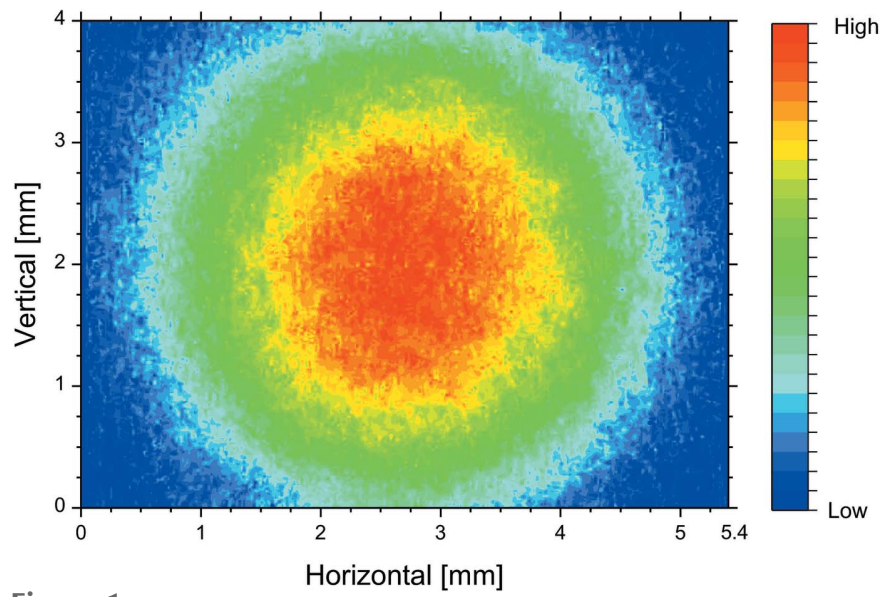

Figure 1

Third-harmonic FEL profile measured using a charge-coupled device camera at a position $0.8 \mathrm{~m}$ away from the downstream cavity mirror. The spot size at the observed position was $2.55 \mathrm{~mm}$, which is consistent with the ideal spot size of $2.40 \mathrm{~mm}$. 
Table 1

Parameters of the optical klystron ETLOK-III and saturated FEL power in the higher-harmonic FEL experiments.

\begin{tabular}{|c|c|c|c|c|c|c|}
\hline $\begin{array}{l}\text { Wavelength } \\
(\mathrm{nm})\end{array}$ & $\begin{array}{l}\text { Cavity } \\
\text { loss }\end{array}$ & $\begin{array}{l}\text { Harmonic } \\
\text { order }\end{array}$ & $\begin{array}{l}K \\
\text { value }\end{array}$ & $n\left(N_{\mathrm{u}}+N_{\mathrm{d}}\right)$ & $\begin{array}{l}\text { Threshold } \\
\text { current }(\mathrm{mA})\end{array}$ & $\begin{array}{l}P_{\text {out }} \text { at } 5 \mathrm{~mA} \\
(\mu \mathrm{W})\end{array}$ \\
\hline \multirow[t]{2}{*}{870} & \multirow[t]{2}{*}{$1.0 \times 10^{-3}$} & Fundamental & 2.09 & 197 & 2.3 & 25 \\
\hline & & Third harmonic & 4.14 & 265 & 3.5 & 4.4 \\
\hline \multirow[t]{3}{*}{890} & \multirow[t]{3}{*}{$4.5 \times 10^{-4}$} & Fundamental & 2.13 & 195 & 0.55 & 320 \\
\hline & & Third harmonic & 4.19 & 269 & 0.90 & 200 \\
\hline & & Fifth harmonic & 5.52 & 357 & 1.5 & 66 \\
\hline \multirow[t]{2}{*}{1530} & \multirow[t]{2}{*}{$1.2 \times 10^{-3}$} & Fundamental & 3.03 & 122 & 1.0 & 310 \\
\hline & & Third harmonic & 5.58 & 209 & 1.6 & 79 \\
\hline
\end{tabular}

oscillations. The solid curves in Fig. 3, which are the calculated dependence using equation (2), are roughly in accord with the measured data. Thus, the bunch lengthening on the higherharmonic FEL oscillation can be also described by equation (2), which is based on the theory of bunch heating for the fundamental FEL oscillation. We demonstrate that the theory of bunch heating is applicable to the higher-harmonic FEL oscillations. tively, and their corresponding values of threshold current were 2.3 and $3.5 \mathrm{~mA}$. As shown in Fig. 2, the bunch lengths on the fundamental and third-harmonic FEL oscillations were different for the same value of single-bunch current. Because the FEL gain for the fundamental FEL is higher compared with that for the third-harmonic FEL, the difference in bunch length with respect to the order of the higher harmonic should be based on investigations of the dependence of the bunch length on the ratio $G_{n} / l_{\mathrm{c}}$, as opposed to the beam current. Fig. 3 shows the measured dependence of the relative bunch length on the ratio in the fundamental and third-harmonic FEL

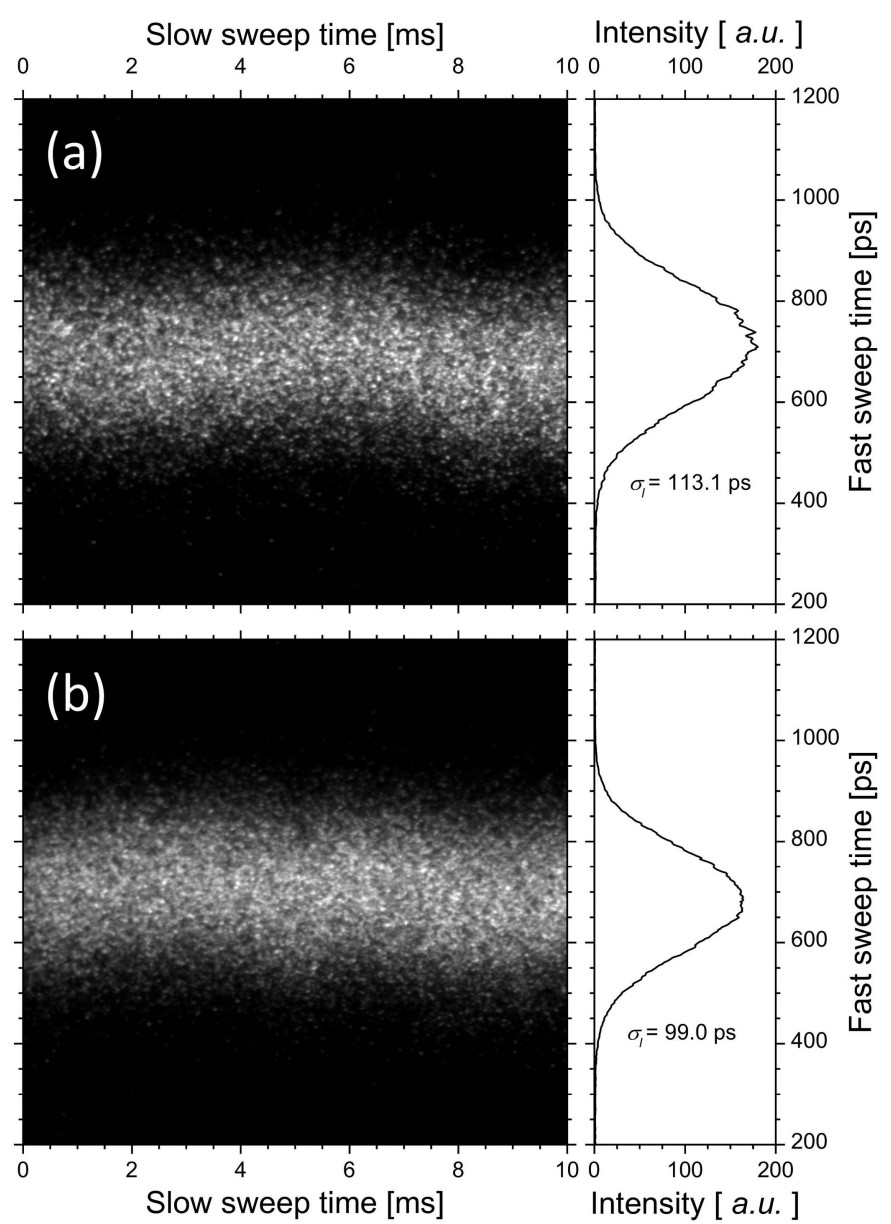

Figure 2

Bunch lengths measured with a dual-sweep streak camera on $(a)$ the fundamental FEL oscillation and $(b)$ the third-harmonic FEL oscillation.

\section{Saturated power of the storage-ring FEL}

An electron beam serves as the laser medium for the FEL interaction, and a FEL oscillation reaches saturation when more energy cannot be extracted from the electron beam. According to the theory of bunch heating extended for the higher-harmonic FEL oscillations, the saturated output power of the storage-ring FEL, $P_{\mathrm{T}}$, has the following relationship with the ratio $G_{n} / l_{\mathrm{c}}$ (Elleaume et al., 1984; Sei et al., 2014a),

$$
P_{\mathrm{T}}=\eta_{\mathrm{c}} \frac{f_{\gamma n}}{2 \pi n\left(N_{\mathrm{u}}+N_{\mathrm{d}}\right)} \ln \left(\frac{G_{n}}{l_{\mathrm{c}}}\right) P_{\mathrm{s}},
$$

where $\eta_{\mathrm{c}}$ is the optical cavity efficiency, which is defined as the quotient of the output mirror transmission divided by the
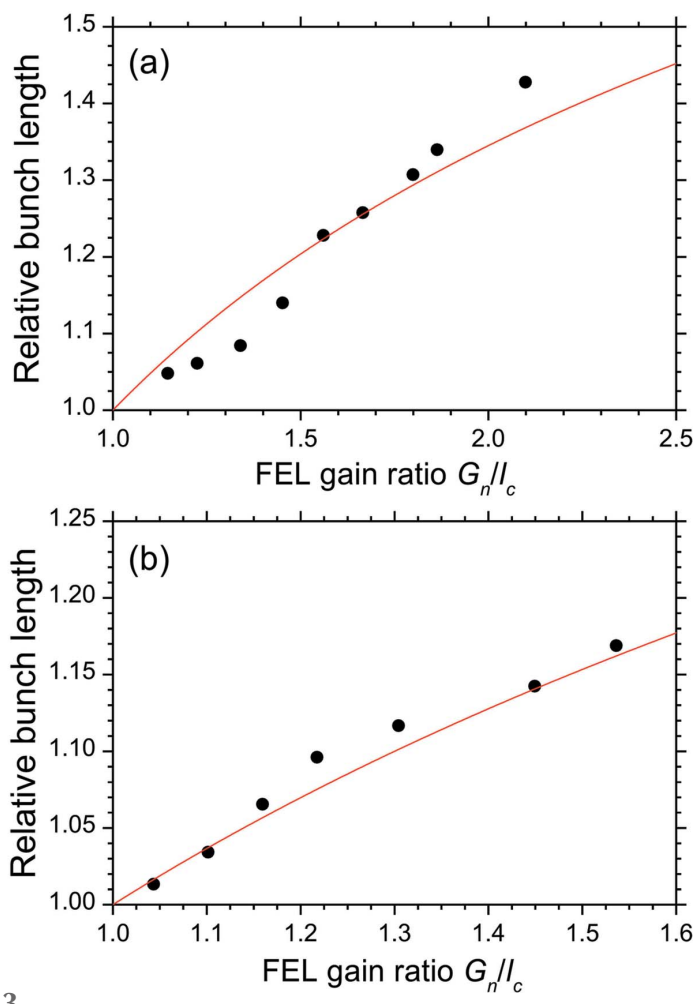

Figure 3

Measured values of the dependence of the relative bunch length on the $G_{n} / l_{\mathrm{c}}$ ratio on $(a)$ the fundamental FEL oscillation and $(b)$ the thirdharmonic FEL oscillation. The solid curves are the calculated dependence values obtained using equation (2). 


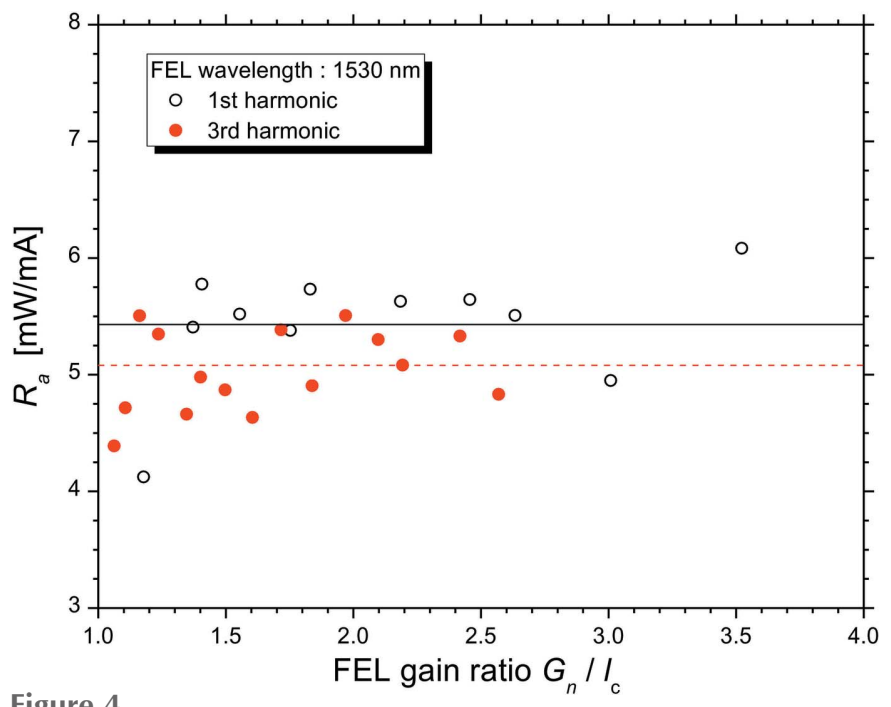

Figure 4

Dependence of the measured ratio $R_{\mathrm{a}}$ on the FEL gain ratio $G_{n} / l_{\mathrm{c}}$ in the fundamental FEL oscillation (open circle) and the third-harmonic FEL oscillation (solid circle). The solid and dotted lines are the average values for the fundamental FEL oscillation and the third-harmonic FEL oscillation, respectively, in the region $G_{n} / l_{\mathrm{c}}>1.1$.

cavity loss. The symbol $P_{\mathrm{s}}$ is the total synchrotron radiation power emitted in the entire storage ring, and it is proportional to the electron-beam current $I_{\mathrm{b}}$. This equation indicates that $P_{\mathrm{T}}$ is a function of the ratio $G_{n} / l_{\mathrm{c}}$, and, subsequently, also of the bunch length on the FEL oscillation. To experimentally confirm that equation (4) holds for any arbitrary order of higher harmonic, we measured the ratio $R_{\mathrm{a}}$ as follows,

$$
R_{\mathrm{a}}=\frac{n\left(N_{\mathrm{u}}+N_{\mathrm{d}}\right)}{I_{\mathrm{b}} f_{\gamma n} \ln \left(G_{n} / l_{\mathrm{c}}\right)} P_{\mathrm{T}} .
$$

The saturated output power was measured using a calibrated power meter (Coherent Inc., OP-2 IR) with the fundamental and the third-harmonic FEL oscillations at a wavelength of $\sim 1530 \mathrm{~nm}$ (Sei et al., 2017). Fig. 4 depicts a plot of the relationship between the measured values of $R_{\mathrm{a}}$ and the $G_{n} / l_{\mathrm{c}}$ ratio. It is noted that the measured values of $R_{\mathrm{a}}$ were roughly constant for both the harmonics in the large area of $G_{n} / l_{\mathrm{c}}$. The mean values of the measured $R_{\mathrm{a}}$ were $5.4 \pm 0.52$ for the fundamental FEL oscillation and $5.1 \pm 0.32$ for the thirdharmonic FEL oscillation, and it was confirmed that they were almost equal. Therefore, the theory of bunch heating is valid for the higher-harmonic oscillation in the storage-ring FEL, and the saturated power of the storage-ring FEL can be described using the effect of bunch lengthening on the higherharmonic oscillation.

\section{Increase of electron-beam lifetime in higher-harmonic FEL oscillations}

Generally, the bunch lengthening increases the lifetime of the electron beam in the storage ring. It has been previously reported that the lifetime was increased by the bunch lengthening on the FEL oscillation. For a low-energy storage ring such as the NIJI-IV, the Touschek effect mainly influences the lifetime. The Touschek lifetime $\tau_{\mathrm{T}}$ can be represented using the momentum acceptance $(\Delta p / p)_{\mathrm{c}}$ by the following equation (Wallén, 2003),

$$
\frac{1}{\tau_{\mathrm{T}}}=\frac{\sqrt{\pi} c r_{\mathrm{e}}^{2} N_{\mathrm{b}}}{\gamma^{3} V_{\mathrm{b}} \sigma_{x}^{\prime}(\Delta p / p)_{\mathrm{c}}^{2}} F\left(\varepsilon_{\mathrm{A}}\right),
$$

where $\gamma, r_{\mathrm{e}}, N_{\mathrm{b}}$ and $\sigma_{x}^{\prime}$ are the energy of the electron expressed in units of its rest energy, the classical electron radius, the number of electrons in a bunch, and the angular divergence of the electron beam in the horizontal plane, respectively. The symbol $V_{\mathrm{b}}$ denotes the volume of the bunch, and it is inversely proportional to the bunch length. The parameter $\varepsilon_{\mathrm{A}}$ is given by

$$
\varepsilon_{\mathrm{A}}=\frac{1}{\left(\gamma \sigma_{x}^{\prime}\right)^{2}}\left(\frac{\Delta p}{p}\right)_{\mathrm{c}}^{2} .
$$

In the case of $\varepsilon_{\mathrm{A}} \ll 1$, the function $F\left(\varepsilon_{\mathrm{A}}\right)$ is approximately given by the following equation,

$$
F\left(\varepsilon_{\mathrm{A}}\right)=-\ln \varepsilon_{\mathrm{A}}-2.077 \text {. }
$$

In the infrared experiments, the horizontal and vertical betatron tunes of the storage-ring NIJI-IV were 2.29 and 1.38, respectively. The horizontal and vertical beam sizes at the centre of the long straight section were measured to be 0.9 and $0.2 \mathrm{~mm}$ at a beam current of $3 \mathrm{~mA}$, respectively. The beam sizes were almost constant in the $2-5 \mathrm{~mA}$ current region. Although the dispersive function was almost zero in the two long straight sections, it increased in the sections containing the bending magnets (Sei et al., 2003). Subsequently, the horizontal beam size and the horizontal angular divergence increased due to energy spreading on the FEL oscillation. The lifetime was also increased owing to other factors besides the bunch lengthening.

To investigate the influence of the order of the higherharmonic FEL oscillation on the lifetime, we measured the electron beam current on the fifth-harmonic FEL oscillation at the wavelength $890 \mathrm{~nm}$ and on the third-harmonic FEL oscillation at the wavelength $1530 \mathrm{~nm}$. As shown in Table 1, the two FEL oscillations could be operated under almost the same conditions as for the $K$ and $N_{\mathrm{d}}$ values. In other words, the electron beam orbit in the third-harmonic FEL experiment was the same as that in the fifth-harmonic FEL experiment. The threshold current in the NIJI-IV infrared FEL system for the third-harmonic FEL oscillation was almost equal to that of its fifth-harmonic. Furthermore, it was possible to observe the influence of the order of the higher-harmonic FEL oscillation on the electron beam at a certain $G_{n} / l_{\mathrm{c}}$ ratio. Fig. 5 shows the decay curves of the electron-beam currents in the presence or absence of the higher-harmonic FEL oscillations. Because the electron beam orbit was set on the central axis of the ETLOKIII, the closed orbit distortion of the electron beam in NIJI-IV increased in the infrared FEL experiments. When the $K$ value was 5.6, the momentum acceptance was approximately $1.5 \times 10^{-3}$. As shown in Fig. 5, the Touschek lifetime was only $9 \mathrm{~min}$ at a electron-beam current of $3.0 \mathrm{~mA}$ in the singlebunch operation. It can be deduced that the lifetime of the 
Table 2

Measured and calculated values of the products of the current and lifetime in the presence and absence of the higher-harmonic FEL oscillations.

\begin{tabular}{|c|c|c|c|c|c|}
\hline \multirow[b]{2}{*}{ Condition } & \multicolumn{3}{|c|}{ Calculated rate of increase of electron-beam lifetime } & \multirow[b]{2}{*}{$\begin{array}{l}\text { Measured product } \\
\text { of lifetime and } \\
\text { current (min } \mathrm{mA})\end{array}$} & \multirow[b]{2}{*}{$\begin{array}{l}\text { Measured rate } \\
\text { of increase of } \\
\text { lifetime }\end{array}$} \\
\hline & $\begin{array}{l}\text { Component of } \\
\text { bunch length }\end{array}$ & $\begin{array}{l}\text { Component of } \\
\text { beam size and } \\
\text { divergence }\end{array}$ & Total & & \\
\hline FEL off & 1 & 1 & 1 & $39.1 \pm 2.4$ & 1 \\
\hline Third-harmonic lasing & 1.37 & 1.11 & 1.52 & $59.7 \pm 7.9$ & $1.53 \pm 0.22$ \\
\hline Fifth-harmonic lasing & 1.19 & 1.05 & 1.25 & $53.6 \pm 3.1$ & $1.34 \pm 0.11$ \\
\hline
\end{tabular}

electron beam increased by oscillating the FEL and its length increased for decreasing order of the higher harmonics. Table 2 shows the values of the products of the current and lifetime in the presence and absence of the higher-harmonic FEL oscillations for a current region of 3.6-3.0 mA. Generally, the product at a storage ring was constant despite the differences in the values of current (Bernardini et al., 1963). The products for the third and the fifth harmonics were, respectively, $1.53 \pm$ 0.22 times and $1.34 \pm 0.11$ times higher than that for the case where the FEL oscillation was absent. Table 2 also shows the products calculated with the electron-beam parameters evaluated by the bunch lengthening on the higher-harmonic FEL oscillations at a current of $3.6 \mathrm{~mA}$. According to the estimation, the calculated products for the third and the fifth harmonics were, respectively, 1.52 times and 1.25 times higher than the case where the FEL oscillation was absent. These are in accord with the measured values. These experimental results suggest that the theory of bunch heating can describe the properties of the electron beam on the higher-harmonic FEL oscillations.

\section{Detuning of the cavity length}

It is known that a macrotemporal structure appears in the storage-ring FEL due to a detuning of the optical cavity

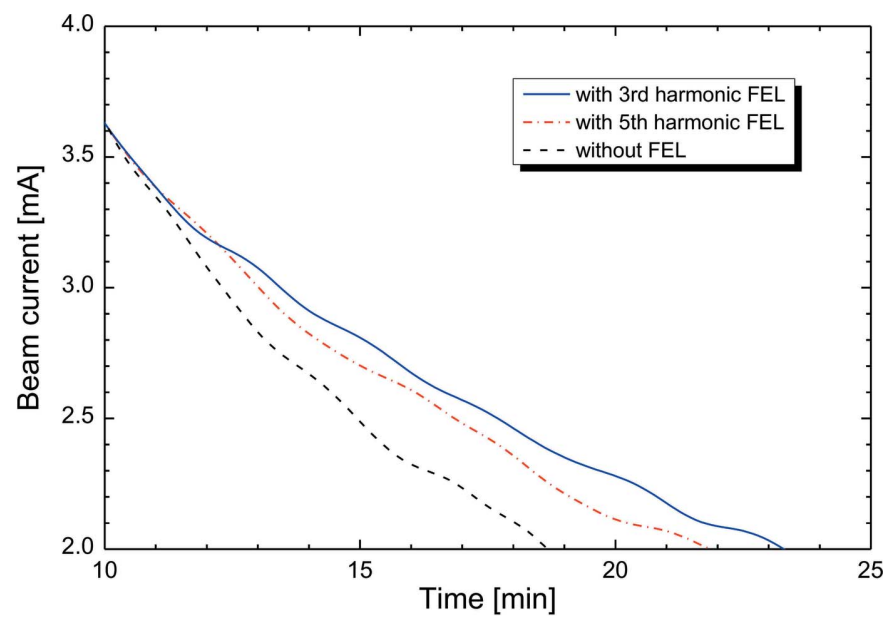

Figure 5

Measured decay curves of the electron-beam current in the presence of the third-harmonic FEL oscillation (solid line) and the fifth-harmonic FEL oscillation (chain line) and in the absence of an FEL oscillation (dotted line). The FEL wavelengths were $1530 \mathrm{~nm}$ for third-harmonic lasing and $890 \mathrm{~nm}$ for fifth-harmonic lasing.
(Elleaume et al., 1984; Couprie et al., 1993; Roux et al., 1997; Litvinenko et al., 2001; Sei et al., 2004). The minute detuning causes period oscillations in the FEL output power and the bunch length, whose typical time scales are from $1 \mathrm{~ms}$ to $100 \mathrm{~ms}$. Such period structures were observed on the higherharmonic FEL oscillations in the NIJI-IV infrared FEL system. However, we are interested in the longer-term behaviour of the FEL output power and the bunch length on the higher-harmonic FEL oscillations. In this paper, we consider time-averaged physical quantities of millisecond order.

Since the FEL gain is proportional to the electron density in the electron bunch, the FEL gain has a Gaussian distribution with a standard deviation equal to the bunch length in the direction of the electron orbit. When the cavity-length detuning is much smaller than the bunch length, the FEL can reach an oscillation even for a low $G_{n} / l_{\mathrm{c}}$ ratio. The bunch length is an essential parameter for the cavity-length detuning curve, which describes a relationship between the detuning length and the FEL intensity. When the maximum FEL gain is fixed, it is anticipated that the FEL intensity at a certain detuning length should be higher as the bunch length is longer. To confirm this estimate, we measured the cavity-length detuning curves on the fifth-harmonic FEL oscillation at $890 \mathrm{~nm}$ and the third-harmonic FEL oscillation at $1530 \mathrm{~nm}$. As mentioned in the last section, these higher-harmonic FELs could oscillate under the same conditions of magnetic field as the ETLOK-III, and the threshold electron-beam currents of these higher-harmonic FELs were approximately equal. Fig. 6 shows the cavity-length detuning curves on two higherharmonic FEL oscillations at a $G_{n} / l_{\text {c }}$ ratio of 2.2. The bunch lengths calculated using equation (2) for the third-harmonic FEL oscillation and the fifth-harmonic FEL oscillation are 122 and $107 \mathrm{ps}$, respectively, at a $G_{n} / l_{\mathrm{c}}$ ratio of 2.2. On the other hand, the full width at half-maximums of the detuning curves, $\Delta D$, were measured to be $1.89 \mu \mathrm{m}$ on the third-harmonic FEL oscillation and $1.28 \mu \mathrm{m}$ on the fifth-harmonic FEL oscillation. These experimental results demonstrated for the first time that the values of $\Delta D$ were different due the difference in the bunch lengths on the FEL oscillations, despite the conditions of the insertion device and the $G_{n} / l_{\mathrm{c}}$ ratio being the same. We could realise such comparisons using the higher-harmonic FEL oscillations.

We experimentally investigate a relationship between the width of the detuning curve and the bunch length on the higher-harmonic FEL oscillation. The detuning can be understood as a gap between the electron bunch and the resonated light pulse in the direction of the electron-beam 

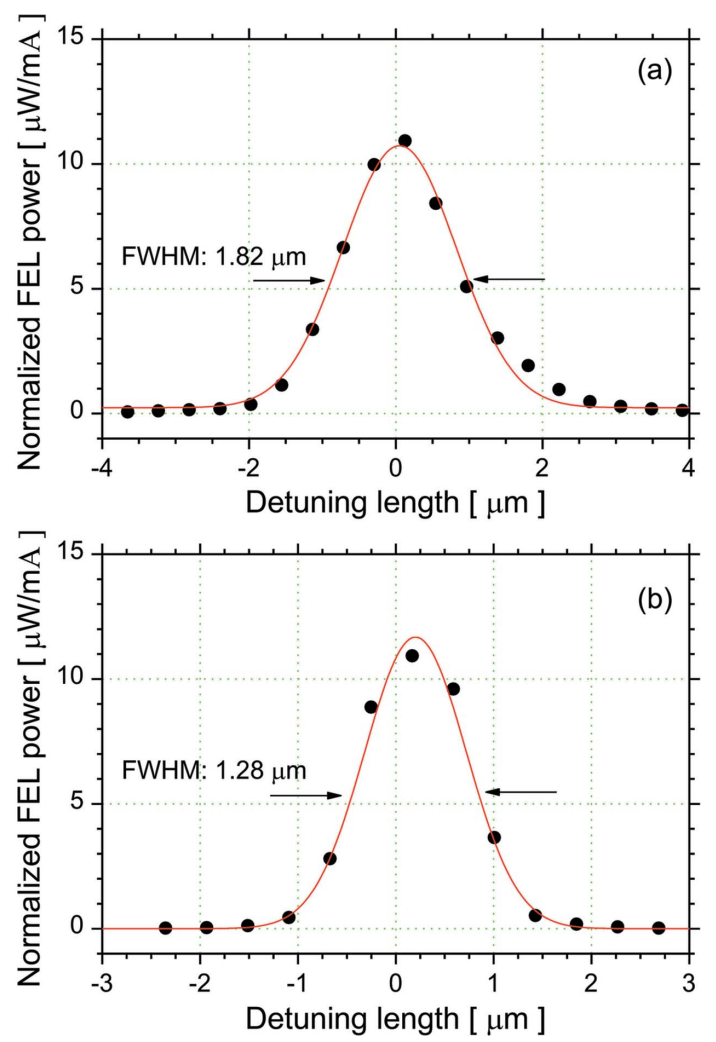

Figure 6

Cavity-length detuning curves for third-harmonic FEL oscillation $(a)$ and fifth-harmonic FEL oscillation (b) at a FEL gain ratio $G_{n} / l_{\mathrm{c}}$ of 2.2. The solid lines are the Gaussian fitting curves of the measured data.

orbit. When a gap exists in the optical cavity, the number of FEL interactions for the light pulse is proportional to the bunch length. The electron bunch has a delay with respect to the light pulse due to its undulating motion in the optical klystron, which is proportional to $n\left(N_{\mathrm{u}}+N_{\mathrm{d}}\right)$ (Deacon et al., 1984). This delay increases the region of the FEL interaction linearly, and is considered to have a positive correlation with $\Delta D$. Moreover, the detuning curve represents the amplification factor of the light pulse caused by the FEL interaction, such that $\Delta D$ would be proportional to the FEL intensity normalized by the electron-beam current, $P_{\mathrm{T}} / I_{\mathrm{b}}$. When $\Delta D$ is much smaller than the bunch length, it is expected that $\Delta D$ is approximately proportional to the product of these parameters $\left[\sigma_{1}, n\left(N_{\mathrm{u}}+N_{\mathrm{d}}\right)\right.$ and $\left.P_{\mathrm{T}} / I_{\mathrm{b}}\right]$, despite the FEL interaction being a nonlinear phenomenon between the electron bunch and the light pulse. Fig. 7 plots the correlation between the product of $n\left(N_{\mathrm{u}}+N_{\mathrm{d}}\right) \sigma_{1} P_{\mathrm{OUT}} / I_{\mathrm{b}}$ and $\Delta D$ in the higherharmonic FEL experiments. The Pearson correlation coefficient between these parameters was evaluated to be 0.9996 for all the higher harmonics. It is noted that the width of the detuning curve was perfectly proportional to the product $n\left(N_{\mathrm{u}}+N_{\mathrm{d}}\right) \sigma_{1} P_{\text {OUT }} / I_{\mathrm{b}}$. Because the FEL intensity is a function of the bunch length given by equations (2) and (4), $\Delta D$ can be directly described in terms of the bunch length. An expression for the width of the detuning curve was determined experimentally, which could be applied to higher-harmonic FEL oscillations.

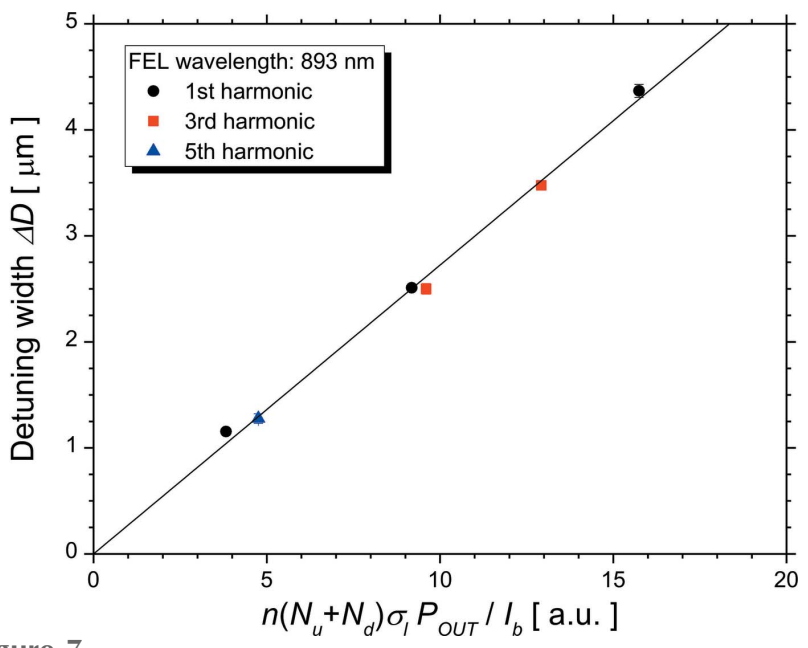

Figure 7

Correlation between $n\left(N_{\mathrm{u}}+N_{\mathrm{d}}\right) \sigma_{\mathrm{l}} P_{\text {OUT }} / I_{\mathrm{b}}$ depending on the bunch length and the width of the cavity-length detuning curve $\Delta D$. The circle, square and triangle symbols are data points for fundamental, thirdharmonic and fifth-harmonic lasing, respectively. The solid line is a fitting line with collinear approximation.

\section{Conclusion}

We developed higher-harmonic FELs using the infrared FEL system in the NIJI-IV storage ring and observed various phenomena caused by the electron bunch on the higherharmonic FEL oscillations. The temporal structure of the electron-beam micropulse was measured by a dual-sweep streak camera, and it was confirmed that bunch lengthening occurred on the higher-harmonic FEL oscillations. The saturated power of the storage-ring FEL was also measured on the higher-harmonic FEL oscillation, and it was demonstrated that the power could be described by the theory of bunch heating. It was observed that the third- and the fifth-harmonic FELs could oscillate for the same conditions of the electron beam and optical klystron by changing the optical cavity mirrors. By adjusting the threshold currents for two higherharmonic FELs to the same value, we could set a condition of the bunch lengths being different for the higher-harmonic FELs despite having the same $G_{n} / l_{\mathrm{c}}$ ratio. The measured lifetimes of the electron beam on the higher-harmonic FELs were affected by the phenomenom of energy spreading. Moreover, we demonstrated that the full width at halfmaximum of the detuning curve was proportional to the product depending on the bunch length in the higherharmonic FEL oscillation.

The generation of the higher-harmonic oscillation is a technique that allows systems of an extreme-ultraviolet (EUV) FEL and X-ray FEL oscillators (XFELO) to have compact designs. It would be possible to develop a compact EUV FEL system using a higher-harmonic resonator in an optical cavity as a seed light. There are few papers that report on the experimental results of the characteristics of the higherharmonic FEL; however, there are hardly any reports on the influence of the higher-harmonic FEL oscillation on the electron beam. We clarified this aspect by measuring the bunch length on the harmonic lasing in the NIJI-IV infrared 
FEL system. We believe that these experimental results will be useful for developing compact EUV FEL and XFELO systems.

\section{Funding information}

The following funding is acknowledged: JSPS KAKENHI (award No. JP16H03912).

\section{References}

Benson, S. V. \& Madey, J. M. J. (1989). Phys. Rev. A, 39, 1579-1581. Bernardini, C., Corazza, G. F., Di Giugno, G., Ghigo, G., Haissinski, J., Marin, P., Querzoli, R. \& Touschek, B. (1963). Phys. Rev. Lett. 10, 407-409.

Billardon, M., Elleaume, P., Ortega, J. M., Bazin, C., Bergher, M., Velghe, M., Deacon, D. A. \& Petroff, Y. (1985). IEEE J. Quantum Electron. 21, 805-823.

Colson, W. B. (1981). IEEE J. Quantum Electron. 17, 1417-1427.

Couprie, M. E., Litvinienko, V., Garzella, D., Delboulbé, A., Velghe, M. \& Billardon, M. (1993). Nucl. Instrum. Methods Phys. Res. A, 331, 37-41.

Dai, J., Deng, H. \& Dai, Z. (2012). Phys. Rev. Lett. 108, 034502.

Deacon, D. A. G., Billardon, M., Elleaume, P., Ortega, J. M., Robinson, K. E., Bazin, C., Bergher, M., Velghe, M., Madey, J. M. J. \& Petroff, Y. (1984). Appl. Phys. B, 34, 207-219.

Deng, H. X. \& Dai, Z. M. (2013). Chin. Phys. C. 37, 102001.

Elleaume, P. (1984a). J. Phys. Fr. 45, 997-1001.

Elleaume, P. (1984b). J. Phys. (Paris), 44(C1), 333-352.

Elleaume, P., Ortéga, J. M., Billardon, M., Bazin, C., Bergher, M., Velghe, M., Petroff, Y., Deacon, D. A. G., Robinson, K. E. \& Madey, J. M. J. (1984). J. Phys. Fr. 45, 989-996.

Hajima, R., Nagai, R., Nishimori, N., Kikuzawa, N. \& Minehara, E. J. (2001). Nucl. Instrum. Methods Phys. Res. A, 475, 43-46.

Jerby, E. \& Gover, A. (1986). Nucl. Instrum. Methods Phys. Res. A, 250, 192-202.

Kato, R., Okuda, S., Nakajima, Y., Kondo, G., Iwase, Y., Kobayashi, H., Suemine, S. \& Isoyama, G. (1998). Nucl. Instrum. Methods Phys. Res. A, 407, 157-160.
Kim, K. J., Shvyd'ko, Y. \& Reiche, S. (2008). Phys. Rev. Lett. 100, 244802.

Litvinenko, V. N., Park, S. H., Pinayev, I. V. \& Wu, Y. (2001). Nucl. Instrum. Methods Phys. Res. A, 475, 240-246.

Neil, G. R., Benson, S. V., Biallas, G., Gubeli, J., Jordan, K., Myers, S. \& Shinn, M. D. (2001). Phys. Rev. Lett. 87, 084801.

Renieri, A. (1979). Nuov. Cim. B, 53, 160-178.

Roux, R., Couprie, M. E., Hara, T., Bakker, R. J., Visentin, B., Billardon, M. \& Roux, J. (1997). Nucl. Instrum. Methods Phys. Res. A, 393, 33-37.

Sei, N., Ogawa, H. \& Okuda, S. (2017). J. Appl. Phys. 121, 023103.

Sei, N., Ogawa, H. \& Yamada, K. (2009). Opt. Lett. 34, 1843-1845.

Sei, N., Ogawa, H. \& Yamada, K. (2010). J. Phys. Soc. Jpn, 79, 093501.

Sei, N., Ogawa, H. \& Yamada, K. (2012a). Appl. Phys. Lett. 101, 144101.

Sei, N., Ogawa, H. \& Yamada, K. (2012b). Opt. Express, 20, 308-316. Sei, N., Ogawa, H. \& Yamada, K. (2012c). J. Phys. Soc. Jpn, 81, 093501.

Sei, N., Ogawa, H. \& Yamada, K. (2014a). JPS Conf. Proc. 1, 014005.

Sei, N., Ogawa, H., Yamada, K., Koike, M. \& Ohgaki, H. (2014b). J. Synchrotron Rad. 21, 654-661.

Sei, N., Ohgaki, H., Mikado, T. \& Yamada, K. (2002). Jpn. J. Appl. Phys. 41, 1595-1601.

Sei, N., Yamada, K. \& Mikado, T. (2004). Jpn. J. Appl. Phys. 43, $577-$ 584.

Sei, N., Yamada, K. \& Ogawa, H. (2008). J. Phys. Soc. Jpn, 77, 074501.

Sei, N., Yamada, K., Ogawa, H., Yasumoto, M. \& Mikado, T. (2003). Jpn. J. Appl. Phys. 42, 5848-5858.

Wallén, E. (2003). Nucl. Instrum. Methods Phys. Res. A, 508, 487495.

Warren, R. W., Haynes, L. C., Feldman, D. W., Stein, W. E. \& Gitomer, S. J. (1990). Nucl. Instrum. Methods Phys. Res. A, 296, 84-88.

Wiedemann, H. (1995). Particle Accelerator Physics II, ch. 10, p. 350. New York: Springer-Verlag.

Wu, Y. K., Benson, S. V., Li, J., Mikhailov, S. F., Neil, G. \& Popov, V. (2008). Proceedings of the 30th International Free Electron Laser Conference, Gyeongju, Korea.

Yamazaki, T., Yamada, K., Sei, N., Ohgaki, H., Sugiyama, S., Suzuki, R., Mikado, T., Noguchi, T., Chiwaki, M., Ohdaira, T. \& Toyokawa, H. (1998). Nucl. Instrum. Methods Phys. Res. B, 144, 83-89. 